EPJ manuscript No.

(will be inserted by the editor)

\title{
Atom-optical elements on micro chips
}

\author{
S. Kraft, A. Günther, P. Wicke, B. Kasch, C. Zimmermann, and J. Fortágh \\ Physikalisches Institut der Universität Tübingen \\ Auf der Morgenstelle 14, 72076 Tübingen, Germany \\ Received: date / Revised version: date
}

\begin{abstract}
We describe the realization of atom-optical elements as magnetic waveguide potentials, beam splitters and gravitational traps on a microchip. The microchip was produced by electroplating gold conductors on an aluminiumoxide substrate. The conductors are 30-150 $\mu \mathrm{m}$ wide and allow for the generation of waveguides at large distances to the chip surface, where surface effects are negligible. We show that these elements can be integrated on a single chip to achieve complex atom-optical circuits.
\end{abstract}

PACS. 03.75.Be Atom and neutron optics - 39.25.+k Atom manipulation - 39.90. $+\mathrm{d}$ Other instrumentation and techniques for atomic and molecular physics

\section{Introduction}

The manipulation of Bose-Einstein condensates by means of microscopic electromagnets [12] has developed into an intensive field of research during the past years. The vision of matter-wave interferometers and the control of single atoms [3] seems within reach by using micron scaled potential structures at a chip surface. Current micro fabrication technologies allow the production of high quality electromagnets which meet the requirements of controlling the motion of atoms on the quantum level. On the other hand, some limitations of atom chips have recently been explored including spin-flip losses near metallic surfaces [4], dispersive atom-surface interactions [5], and technical limitations such as geometrical imperfections of micro electromagnets [6 7]. As important as micron scaled potential barriers are smooth waveguide potentials in atomoptical circuits. These enable guiding and splitting of matterwaves, similar to the manipulation of photons in optical fibers and beam splitters. It is preferable to form such waveguides relatively far from the chip surface where the cloud is less affected by surface effects.

In this article, we describe magnetic waveguides, beam splitters, and gravitational traps. These atom-optical elements are all implemented on a single chip (Fig. 11) currently used in our groups, which is routinely loaded with Bose-Einstein condensates [8]. It has been produced by electroplating a $6 \mu \mathrm{m}$ thick gold layer on a $250 \mu \mathrm{m}$ thick aluminium-oxide substrate. The minimal width and spacing of the conductors is $30 \mu \mathrm{m}$. The chip consists of three conductors with a constant width of 100 $\mu \mathrm{m}(\mathrm{QP} 1-\mathrm{QP} 3)$ and nine additional conductors with varying width $(\mathrm{G} i)$. Perpendicular conductors on the back side of the chip allow positioning of the trap parallel to the conductors QP [8]. Experimentally, we found that in a pulsed duty cycle (3s operation time in $60 \mathrm{~s}$ cycle time) the $100 \mu \mathrm{m}$ and $30 \mu \mathrm{m}$ wide conductors can carry a current up to $2 \mathrm{~A}$ and $1 \mathrm{~A}$, respectively. An atomic cloud, initially confined in a waveguide potential

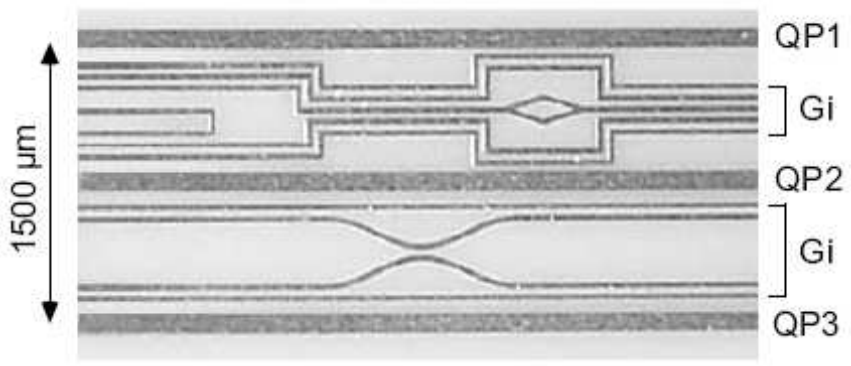

Fig. 1. Complex atom-optical circuit. Waveguide and interferometer geometries as well as gravitational traps are arranged between QP1QP2 and QP2-QP3. The QP conductors define a central waveguide from which neighboring microtraps can be loaded adiabatically.

above QP2 can be adiabatically loaded into the mictrotraps between QP1 and QP2, as well as between QP2 and QP3. Neighboring traps can exchange atoms directly, more distant traps are connected via waveguides.

\section{Highly elongated traps}

A simple waveguide is formed by the circular field of a thin current carrying conductor superimposed by a homogeneous bias field [9 10 11]. The two fields compensate each other along a line parallel to the conductor. Centered around this line, the magnetic field is well approximated by a two dimensional quadrupole, and paramagnetic atoms in a low field seeking state become trapped in radial direction. The quadrupole channel is characterized by the gradient of the magnetic field in the radial direction $a_{\mathrm{r}}$. If an additional magnetic offset field $B_{\text {off }}$ is applied parallel to the waveguide [12], the radial confinement 


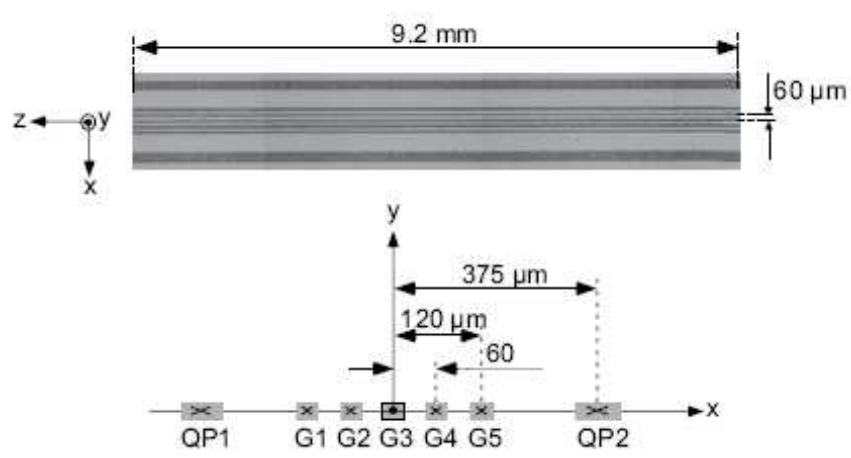

Fig. 2. Waveguide with parallel conductors on a chip. (Top) Microscope image of the chip. (Bottom) Conductor geometry. If the center conductor G3 is driven with a current opposite in direction to the outer conductors (QP1, G1, G2, G4, G5 and QP2), a waveguide potential forms. With pair wise equal currents in QP1-QP2, G1-G5, and G2-G4, the waveguide forms above G3.

becomes harmonic, characterized by the radial oscillation frequency $\omega_{\mathrm{r}}=a_{\mathrm{r}} \cdot \sqrt{g_{\mathrm{F}} \mu_{\mathrm{B}} \mathrm{m}_{\mathrm{F}} /\left(m B_{\mathrm{off}}\right)}$, with the Landé factor $g_{\mathrm{F}}$, the mass of the atom $m$, and the Bohr magneton $\mu_{\mathrm{B}}$. In this article, the trap frequencies are calculated for ${ }^{87} \mathrm{Rb}$ atoms in the $\mathrm{F}=2, \mathrm{~m}_{\mathrm{F}}=2$ hyperfine ground state.

\subsection{Waveguides with parallel wires}

A waveguide potential with parallel on-chip conductors has been demonstrated [13]. Here, we describe the realization of a waveguide which allows increasing the radial compression by using additional wires for the bias field. The section of the chip used to build the waveguide consists of 7 parallel conductors (Fig. 22. Five conductors of the width $30 \mu \mathrm{m}$ are in the center area (G1 - G5) with centers separated by $60 \mu \mathrm{m}$. Another two, wider conductors QP1 and QP2 (100 $\mu$ m width) complete the setup. The centers of these outer conductors are $375 \mu \mathrm{m}$ from the conductor G3 in the middle. The current of G3 is opposite in direction to the other conductors. The waveguide is centered above G3 by pair wise using the same currents: $I_{\mathrm{G} 2}=I_{\mathrm{G} 4}$, $I_{\mathrm{G} 1}=I_{\mathrm{G} 5}$ and $I_{\mathrm{QP} 1}=I_{\mathrm{QP} 2}$. The circular magnetic field of the center conductor is then superposed by the bias field arising from the outer conductors. In this geometry the radial gradient is not only due to the gradient of the central conductor but also due to a gradient of the inhomogeneous bias field.

For simplification, let us first assume a waveguide achieved by the three conductors G2, G3, and G4 of Fig. 2 The separation between the middle of the conductors is $d$. The magnetic field on the y axis of this configuration is given by the sum of the fields of the central conductor and of the outer conductors

$$
B(x=0, y)=-\frac{\mu_{0}}{2 \pi} \frac{I_{\mathrm{C}}}{y}+\frac{\mu_{0}}{\pi} \frac{I_{\mathrm{O}} y}{d^{2}+y^{2}},
$$

with $I_{\mathrm{C}}$ the current in the central conductor $\mathrm{G} 3$ and $I_{\mathrm{O}}$ the current in each of the outer conductors G2 and G4. The waveguide formes at a distance $y_{0}$ to the surface:

$$
y_{0}=d \sqrt{\frac{I_{\mathrm{C}}}{2 I_{\mathrm{O}}-I_{\mathrm{C}}}} .
$$

\begin{tabular}{|c|c|c|c|c|c|c|}
\hline $\begin{array}{c}y_{0} \\
{[\mu \mathrm{m}]}\end{array}$ & $\begin{array}{l}I_{\mathrm{QP}} \\
{[\mathrm{A}]}\end{array}$ & $\begin{array}{c}I_{\mathrm{G} 1, \mathrm{G} 5} \\
{[\mathrm{~A}]}\end{array}$ & $\begin{array}{c}I_{\mathrm{G} 2, \mathrm{G} 4} \\
{[\mathrm{~A}]}\end{array}$ & $\begin{array}{l}I_{\mathrm{G} 3} \\
{[\mathrm{~A}]}\end{array}$ & $\begin{array}{c}a_{\mathrm{r}} \\
{[\mathrm{T} / \mathrm{m}]}\end{array}$ & $\begin{array}{c}\nu_{\mathrm{r}} \\
{[\mathrm{kHz}]}\end{array}$ \\
\hline 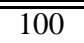 & 2 & 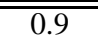 & $\overline{\overline{0}}$ & $\bar{~} 1$ & 27 & $\begin{array}{l}3.5 \\
\end{array}$ \\
\hline 90 & 2 & 1 & 0.044 & 1 & 34 & 4.3 \\
\hline 80 & 2 & 1 & 0.164 & 1 & 42 & 5.3 \\
\hline 70 & 2 & 1 & 0.31 & 1 & 54 & 6.9 \\
\hline 60 & 2 & 1 & 0.5 & 1 & 74 & 9.4 \\
\hline 50 & 2 & 1 & 0.773 & 1 & 111 & 14.1 \\
\hline 40 & 2 & 1 & 1 & 0.861 & 163 & 20.7 \\
\hline 30 & 2 & 1 & 1 & 0.544 & 203 & 25.8 \\
\hline 20 & 2 & 1 & 1 & 0.266 & 244 & 31 \\
\hline 10 & 2 & 1 & 1 & 0.071 & 277 & 35.2 \\
\hline
\end{tabular}

Table 1. Gradients and radial oscillation frequencies of the waveguide (Fig. 2) for different distances $y_{0}$ above the central conductor G3. To calculate the trap frequencies, a homogeneous offset field of $1 \mathrm{G}$ along the waveguide was assumed.

The radial gradient $a_{\mathrm{r}}$ is given by the derivative of the magnetic field at this point. In terms of the current in the outer conductors $I_{\mathrm{O}}$ and the distance of the trap center to the chip $y_{0}$ the gradient is given by

$$
a_{\mathrm{r}}\left(I_{\mathrm{O}}, y_{0}\right)=\frac{\mu_{0}}{\pi} \frac{I_{\mathrm{O}}}{d^{2}} \cdot \frac{1}{\left(1+\left(y_{0} / d\right)^{2}\right)^{2}} .
$$

There are two ways to increase the gradient. One is to increase the current in the outer conductors $I_{\mathrm{O}}$. As this reduces $y_{0}$, it increases the gradient stronger than linearly. However, the flow of the dissipated heat sets an upper limit $I_{\mathrm{O}, \max }$ for this current. The other way is to lower $y_{0}$ by decreasing the current in the central conductor $I_{\mathrm{C}}$. At $I_{\mathrm{C}}=0$, the maximal possible gradient of

$$
a_{\mathrm{r}, \max }=4 \frac{\mu_{0}}{2 \pi} \frac{I_{\mathrm{O}, \max }}{d^{2}}
$$

is reached, in the limit of touching the conductor. This corresponds to a waveguide build only by two wires with currents in the same direction [14].

In the setup shown in Figure 2, additional pairs of wires contribute to the bias field. The increased bias field allows higher current in the central conductor and hence increases the maximal possible gradient at the position of the trap. For the sevenwire configuration and with currents of 1A in G1, G2, G4, G5 and $2 \mathrm{~A}$ in QP1, QP2, we calculate a radial gradient of $a_{\mathrm{r}}\left(y_{0}=0\right)=289 \mathrm{Tm}^{-1}$. With an axial offset field of $1 \mathrm{G}$, the radial oscillation frequency is $\omega_{\mathrm{r}}\left(y_{0}=0\right)=2 \pi \cdot 36.8 \mathrm{kHz}$. Table 1 shows typical values for different currents in the conductors. Note, that the currents in the conductors producing the bias field are not driven equally. For traps near to the surface, a small distance of the conductors generating the bias field to the central conductor is favorable, while for traps far from the surface, a bigger distance is preferable. More conductors increase the flexibility for choosing the position of the bias field generating elements.

\subsection{Folded waveguide configuration}

A chip with a set of parallel conductors as in Fig. 2 allows tight radial confinement even with moderate currents. However, the 


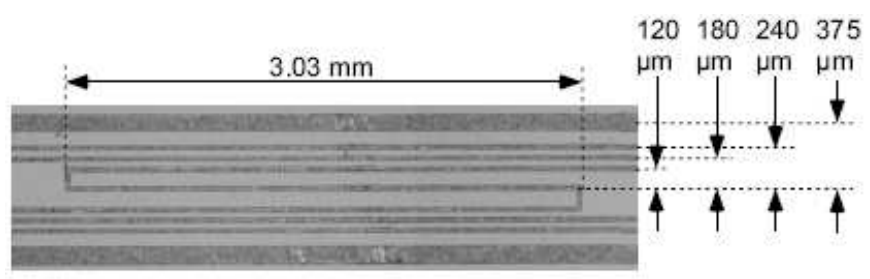

Fig. 3. In the folded alinement, the circular magnetic field of the central conductor generates its own bias field. This adds to the compression due to the outer conductors and increases the confinement.

\begin{tabular}{|c|c|c|c|c|c|c|}
\hline $\begin{array}{c}y_{0} \\
{[\mu \mathrm{m}]}\end{array}$ & $\begin{array}{l}I_{\mathrm{QP}} \\
{[\mathrm{A}]} \\
\end{array}$ & $\begin{array}{c}I_{\mathrm{G} 1, \mathrm{G} 5} \\
{[\mathrm{~A}]}\end{array}$ & $\begin{array}{c}I_{\mathrm{G} 2, \mathrm{G} 4} \\
{[\mathrm{~A}]}\end{array}$ & $\begin{array}{l}I_{\mathrm{G} 3} \\
{[\mathrm{~A}]}\end{array}$ & $\begin{array}{c}a_{\mathrm{r}} \\
{[\mathrm{T} / \mathrm{m}]}\end{array}$ & $\begin{array}{c}\nu_{\mathrm{r}} \\
{[\mathrm{kHz}]}\end{array}$ \\
\hline 100 & 1.35 & $\overline{0}$ & 0 & 1 & 26 & 3.3 \\
\hline 90 & 2 & 0.25 & 0 & 1 & 36 & 4.5 \\
\hline 80 & 2 & 1 & 0.03 & 1 & 49 & 6.2 \\
\hline 70 & 2 & 1 & 0.764 & 1 & 68 & 8.6 \\
\hline 60 & 2 & 1 & 1 & 0.696 & 68 & 8.6 \\
\hline 50 & 2 & 1 & 1 & 0.421 & 62 & 7.9 \\
\hline 40 & 2 & 1 & 1 & 0.242 & 58 & 7.3 \\
\hline 30 & 2 & 1 & 1 & 0.125 & 54 & 6.9 \\
\hline 20 & 2 & 1 & 1 & 0.0525 & 52 & 6.6 \\
\hline 10 & 2 & 1 & 1 & 0.0128 & 48 & 6.1 \\
\hline
\end{tabular}

Table 2. Gradients and radial oscillation frequencies of the waveguide produced by a folded wire configuration (Fig. 3 for different distances $y_{0}$ above the central conductor G3. To calculate the trap frequencies, a homogeneous offset field of $1 \mathrm{G}$ along the waveguide was assumed.

waveguide is located at a rather small distance to the chip surface. Imperfections of the conductor geometry may thus make the potential of the waveguide irregular leading to undesired fragmentation of atomic clouds [6 7].

To achieve a smooth waveguide potential a large surface - waveguide distance is preferable. When the total number of conductors is fixed a folded wire configuration (Fig. 3 can increase the radial gradient at certain distances. In this setup, the folded upper and lower parts of the central conductor contribute to the bias field of the outer conductors. The contribution becomes relevant for currents in G3 which are comparable to the current in the outer conductors and is negligible for smaller currents. For small currents, i.e. traps near the surface of the chip, the maximum achievable compression and gradient are reduced compared to the previous setup (Fig. 2) because the conductors generating the bias field are further away from the center. Table 2 shows gradients and trap frequencies for the same set of distances as in table 1 . The variation of the gradient with the distance is reduced compared to the setup with straight conductors. At working distances of $70-100 \mu \mathrm{m}$, however, the gradient is larger in this configuration. At larger distances the solution with straight conductors produces larger trap frequencies. This is because in the folded geometry the inner conductors contribute to the bias field. For large distances it would be preferable to place the conductors generating the bias field further from the middle conductor.

Comparing the two geometries shows that folding the central conductor increases the radial confinement at certain distances. As part of the bias field is always produced by the inner a)

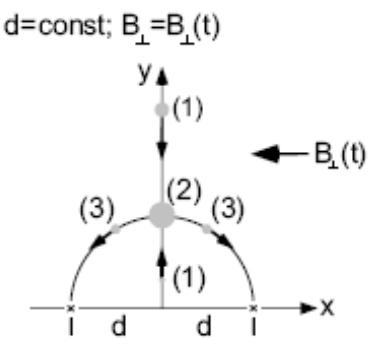

b) $d=d(t) ; B_{\perp}=$ const

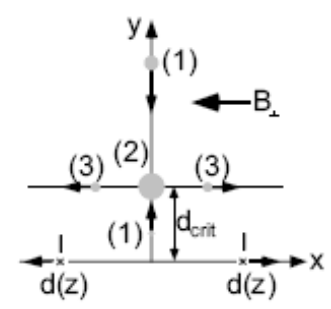

Fig. 4. Beam splitter geometries. a) Beam splitting by changing the bias field above two conductors separated by a constant distance. The trajectories of quadrupole waveguides is shown while the bias field changes. b) Beam splitting with constant bias field and changing the distance between the conductors. The trajectories of the waveguide potentials are shown as explained in the text.

conductor one looses flexibility in choosing the field generating elements as freely as in the setup with straight conductors. This leads to a smaller bias field outside of the ideal distance range.

\section{Beam splitters}

A more complex atom optical element than a waveguide is a beam splitter [11], in which the atomic matter wave is divided into two parts. In atom-optical circuits, the divided matterwave would propagate in separate waveguides before an inverse beam splitter would recombine them producing interference.

Fig. 4 shows two different principles to realize a beam splitter. Both rely on two wires driven with currents in the same direction and a homogeneous bias field $B_{\perp}$. The chip surface is assumed to be horizontal with the gravity parallel to the surface vector.

\subsection{Beam splitters based on a varying bias field}

The field of two parallel conductors combined with a variable bias field can be used for splitting an atomic wave function [15]. Fig. 4 a) illustrates the geometry and the trajectories of waveguide potentials for a varying bias field. Initially, in a small bias field (1), two quadrupole waveguides are located on the $y$-axis. While the bias field is increased, the two waveguides approach each other. At a critical bias field $B_{\text {crit }}=\frac{\mu_{0}}{2 \pi} \frac{I}{d}$ they merge to a hexapole (2) at a distance $d$ to the surface of the chip. The distance $d$ is half of the separation between the two conductors. Further increase of the bias field splits the waveguide into another two quadrupole waveguides. They move along a half circle to the position of the conductors (3).

The realization of such a beam splitter in our setup is shown in Fig. 2 The beam splitter geometry is achieved by applying currents in G2 and G4 as well as in QP1 and QP2 for generating the bias field. When the current in these conductor is increased the bias field increases. 


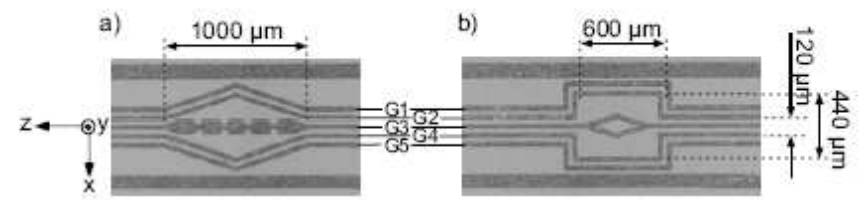

Fig. 5. Realization of beam splitters (and inverse beam splitters) on a chip. a) Interferometers are defined by the pairs of conductors G1+G5 and $\mathrm{G} 2+\mathrm{G} 4$. The bias field can be generated by QP1 and QP2. In b) the conductors G1,G2, G4 and G5 can be used for beam splitters. The separation of the waveguides is faster then in a) due to the instantaneous increase of the distance of the conductors. The conductor (G3) and a bias field generated by the surrounding conductors define another interferometer.

\subsection{Beam splitters based on varying the distance between the conductors}

Another possible implementation of a beam splitter is shown in Fig. (4)b). The bias field stays constant while the distance $d(z)$ between the conductores varies [16].

If the distance $d$ of the two conductors to the y-axis is smaller than a critical distance $d_{\text {crit }}=\frac{\mu_{0}}{2 \pi} \frac{I}{B_{\perp}}$ (1), the two waveguides lie on the y-axis ( $I$ is the current in both of the conductors). As the distance between the conductors increases, the waveguides merge vertically into a single hexapole waveguide at the distance $d_{\text {crit }}$ from the surface (2). Further increasing the distance of the conductors splits the waveguides into two quadrupoles which separate at constant height above the chip surface.

The setup introduced in this article includes multiple onchip beam splitters with varying distance. Examples are shown in Fig. 5 The conductor geometry defines the separation, trajectories and finally the area of the interferometer, enclosed by the waveguides. The bias field can either be generated by external coils or by the conductors QP1 and QP2. The setup in a) consists of two interferometers using either the pair G1 + G5, or G2 + G4 and a bias field. In b) the conductors G1 + G5 and $\mathrm{G} 2+\mathrm{G} 4$ form interferometers with the same maximal separation of the atoms. However, due to the rapid increase of the distance of the conductors the waveguides separate faster. The conductor G3 realizes another interferometer. Here, the current splits into two branches and is recombined at a further position. Unbalance may occur if the current does not split up equally.

Fig. [ shows trajectories of the waveguide center for an interferometer formed by the conductors G2 and G4 in Fig. [5b). The current in each conductor is $0.5 \mathrm{~A}$. A bias field of $5 \mathrm{G}$ is applied. Fig. 6 a) shows a projection onto the y-z plane. Due to gravity, after recombination a condensate will choose the lower branch for $z<-100 \mu \mathrm{m}$ and $z>100 \mu \mathrm{m}$.

Although the micro fabricated conductor pattern provides a well defined geometry and precise control over magnetic fields, the operation of these beam splitters is sensitive to ambient magnetic stray fields. A magnetic field along the $y$-axis changes the trajectories such that merging of the quadrupole waveguides is inhibited. Instead, they pass each other. The adjustment of the beam splitters may thus be nontrivial.
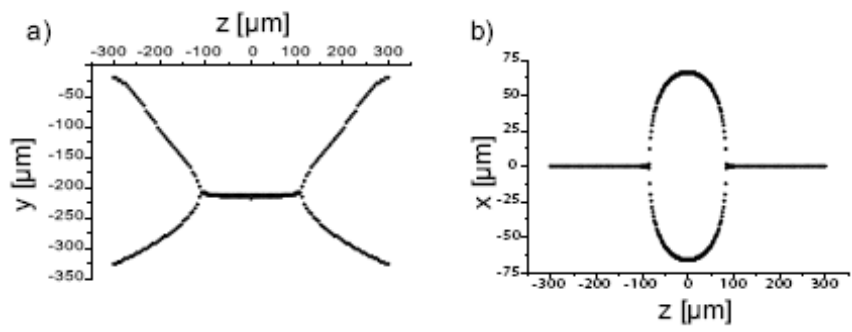

Fig. 6. Trajectories of the waveguide center for the interferometer shown in Fig. 5b. The current in the conductors G2 and G4 is $I_{\mathrm{G} 2}=$ $I_{\mathrm{G} 4}=0.5 \mathrm{~A}$. An additional homogeneous bias field of $B_{\perp}=5 \mathrm{G}$ is applied. a) and b) show projection onto the $y-z$ and $x-z$ planes, respectively.

\section{Gravitational traps}

Let us consider the beam splitter with constant bias field and varying distance between the conductors (section 3.2). The variation of the distance between the conductors, as long as it is smaller than the critical distance, only changes the height of the waveguides above the chip. Provided that the chip is mounted horizontally and the experiments are performed in gravity, a gravitational potential arises. Potential wells and hills along the waveguide can be achieved by changing the conductor geometry.

\subsection{Two wires with changing separation}

a)
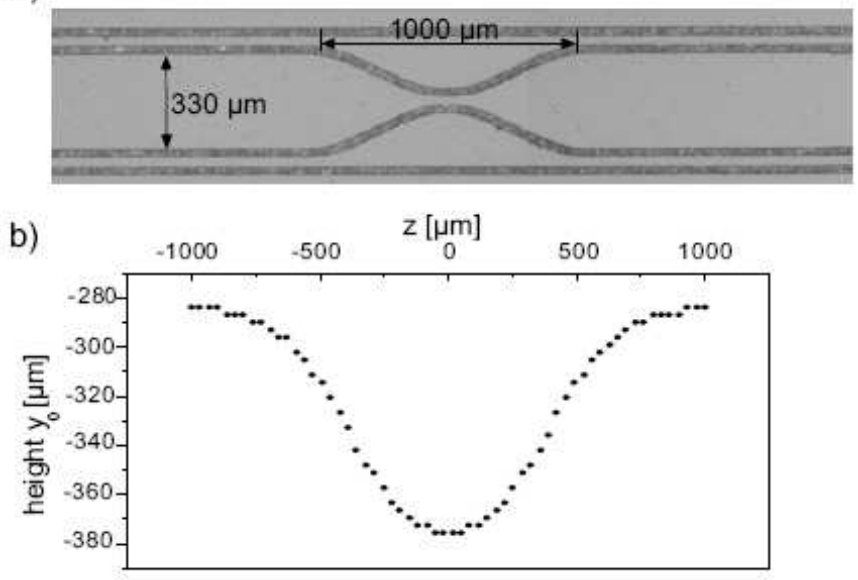

Fig. 7. Gravitational trap. a) Implementation of a gravitational trap for cold atoms on a chip. The magnetic field of the curved conductors in a bias field form a waveguide with varying height. b) Potential for a current of $1 \mathrm{~A}$ in the inner conductors and a homogeneous bias field of $10 \mathrm{G}$.

A waveguide with a gravitational axial confinement can be realized by two curved conductors and a bias field (Fig. [7). The 
a) geometry of the conductor
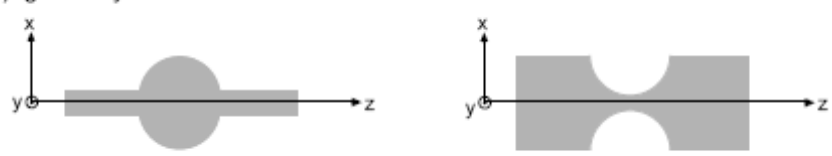

b) position of the waveguide
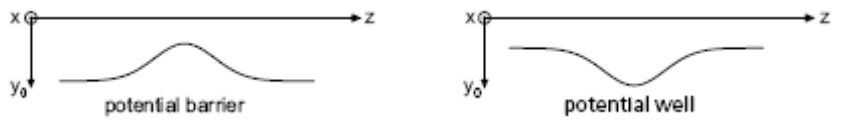

Fig. 8. Sketch of miniaturized gravitational traps. Broadening the conductor decreases the distance of the waveguide to the surface, narrowing increases it. In gravity, the axial potential of a waveguide exhibits gravitational barriers and wells.

waveguide forms with varying distance to the chip surface. Calculating the potential for a current of $1 \mathrm{~A}$ in the curved conductors and a bias field of $10 \mathrm{G}$ (Fig. 7 b) yields that around its minimum $z=0$, the potential can be approximated by a parabola with a curvature of $b=521 \mathrm{Tm}^{-2}$. The height variation of $94 \mu \mathrm{m}$ leads to a trap depth of $9.6 \mu \mathrm{K}$, sufficient to trap a condensate or a thermal cloud near the critical temperature. The overall distance of more than $300 \mu \mathrm{m}$ to the chip surface assures that the potential of the waveguide is not influenced by imperfections of the conductors. A condensate could thus oscillate nearly unperturbed in such a trap. The oscillation frequency can be calculated by considering the potential energy:

$$
U=m g h=\frac{1}{2} m g b x^{2}=\frac{1}{2} k x^{2}
$$

with $k=m g b$. This leads to an harmonic oscillation with the frequency

$$
\omega=\sqrt{\frac{k}{m}}=\sqrt{g b} .
$$

The oscillation frequency with the parameters given above is $\omega=2 \pi \cdot 11.4 \mathrm{~Hz}$. These kind of traps could find use in measurements of gravity since the oscillation frequency of a condensate can be measured with high accuracy [17].

\subsection{One wire with changing width}

Gravitational traps can be miniaturized by changing the width of the conductor which is used for generating the waveguide. The geometry of conductors for potential wells and barriers, as well as the corresponding gravitational potentials, are plotted in Fig. 8. For the entire potential experienced by a cloud of paramagnetic atoms, the magnetic field components due to changes in the conductor geometry have to be taken into account.

\section{Conclusion}

We have demonstrated the fabrication of magnetic wave guides for atoms with gold conductors on an aluminium oxide substrate. By using a set of parallel conductors the confinement can be as high as in standard optical traps even for large distances from the surface where unwanted surface effects can be neglected. On the same chip also spatial beam splitter geometries have been realized. Finally, gravitational traps are demonstrated with an axial oscillation frequency proportional to the square root of the gravitational constant $\mathrm{g}$. Condensates in such traps offer novel perspectives for the construction of gravimeters since they oscillate practically frictionless with a very high Q-factor.

This work was supported by the Deutsche Forschungsgemeinschaft, Landesstiftung Baden-Württemberg, and EU MarieCurie RTN on Atom Chips.

\section{References}

1. H. Ott, J. Fortágh, G. Schlotterbeck, A. Grossmann, and C. Zimmermann. Bose-Einstein Condensation in a Surface Microtrap. Phys. Rev. Lett. 87, 230401 (2001).

2. W. Hänsel, P. Hommelhoff, T. Hänsch, and J. Reichel. BoseEinstein condensation on a microelectronic chip. Nature 413, 498 (2001).

3. U. Dorner, P. Fedichev, D. Jaksch, M. Lewenstein, and P. Zoller. Entangling strings of neutral atoms in 1D atomic pipeline structures. Phys. Rev. Lett. 91, 073601 (2003).

4. C. Henkel and M. Wilkens. Heating of trapped atoms near thermal surfaces. Europhys. Lett. 47, 414 (1999).

5. Y. Lin, I. Teper, C. Chin, and V. Vuletić. Impact of the CasimirPolder potential and Johnson noise on Bose-Einstein condensate stability near surfaces. Phys. Rev. Lett. 92, 050404 (2004).

6. S. Kraft, A. Günther, H. Ott, D. Wharam, C. Zimmermann, and J. Fortágh. Anomalous longitudinal magnetic field near the surface of copper conductors. J. Phys. B 35, L469 (2002).

7. J. Esteve, D. Stevens, C. Aussibal, T. Schumm, C. Figl, D. Mailly, I. Bouchoule, C.I. Westbrook, A. Aspect. Role of wie imperfections in micromagnetic traps for atoms. Phys Rev A 70, 043629 (2004).

8. A. Günther, M. Kemmler, S. Kraft, C. J. Vale, C. Zimmermann, J. Fortágh. Combined chips for atom optics. PRA in press (2005)

9. J. Fortágh, A. Grossmann, C. Zimmermann, and T. Hänsch. Miniaturized wire trap for neutral atoms. Phys. Rev. Lett. 81, 5310 (1998).

10. J. Reichel. Microchip traps and Bose-Einstein condensation. Appl. Phys. B 74, 469 (2002).

11. R. Folman, P. Krüger, J. Schmiedmayer, J. Denschlag, and C. Henkel. Microscopic atom optics: From wires to an atom chip. Adv. At. Mol. Opt. Phys. 48, 263 (2002).

12. D. E. Pritchard. Cooling Neutral Atoms in a Magnetic Trap for Precision Spectroscopy. Phys. Rev. Lett. 51, 1336 (1983).

13. N. Dekker, C. Lee, V. Lorent, J. Thywissen, S. Smith, M. Drndic, R. Westervelt, and M. Prentiss. Guiding neutral atoms on a chip. Phys. Rev. Lett. 84, 1124 (2000).

14. J. Thywissen, M. Olshanii, G. Zabow, M. Drndic, K. Johnson, R. Westervelt, and M. Prentiss. Microfabricated magnetic waveguides for neutral atoms. Eur. Phys. J. D 7, 361 (1999).

15. E. Hinds, C. Vale, and M. Boshier. Two-wire waveguide and interferometer for cold atoms. Phys. Rev. Lett. 86, 1462 (2001).

16. D. Cassettari, B. Hessmo, R. Folman, T. Maier, and J. Schmiedmayer. Beam splitter for guided atoms. Phys. Rev. Lett. 85, 5483 (2000).

17. H. Ott, J. Fortágh, S. Kraft, A. Günther, D. Komma, and C. Zimmermann. Nonlinear dynamics of a Bose-Einstein condensate in a magnetic waveguide. Phys. Rev. Lett. 91, 040402 (2003). 